\title{
MULTICHANNEL SAMPLING OF PARAMETRIC SIGNALS WITH A SUCCESSIVE APPROXIMATION PROPERTY
}

\author{
Julius Kusuma and Vivek K Goyal \\ Massachusetts Institute of Technology \\ Signal Transformation and Information Representation Group
}

\begin{abstract}
Recently the sampling theory for certain parametric signals based on rate of innovation has been extended to all sampling kernels that satisfy the Strang-Fix conditions, thus including many attractive choices with finite support. We propose a new sampling scheme in which samples are taken simultaneously at the outputs of multiple channels. This new scheme is closely related to previously known cases, but provides a successive approximation property that can be used for detecting undermodeling. We also draw connections to splines and multi-scale sampling of signals.
\end{abstract}

Index Terms - Signal sampling, Sampling methods, Delay estimation, Signal detection, Signal reconstruction

\section{INTRODUCTION}

Recent work has shown that it is possible to obtain perfect reconstruction of certain signals with a finite number of degrees of freedom per unit time-Finite Rate of Innovation (FRI) - upon filtering and uniform sampling at close to the rate of innovation [1]. This extends elementary sampling theory to parametric signal classes, removing the importance of Fourier bandwidth. For example, consider the class of signals described by

$$
x(t)=\sum_{k} c_{k} \operatorname{sinc}(t / T-k), \quad\left(c_{k}\right) \in \ell^{2}(\mathbb{Z}) .
$$

Because of properties of the sinc function, we know that this is the set of bandlimited functions in $\mathcal{L}^{2}(\mathbb{R})$ that have Nyquist rate of $1 / T$ samples per second. Heuristically, we can observe that the signal is described by one $c_{k}$ per $T$ seconds, so the ability to reconstruct from samples does not depend on the sinc function or Fourier bandwidth. Rate of innovation is the formalization of this intuition.

Now consider the class of signals given by

$$
x(t)=\sum_{k=0}^{K-1} c_{k} \delta\left(t-t_{k}\right)
$$

This work was supported in part by the Texas Instruments Leadership University Consortium Program.
These signals are not bandlimited. However, any signal can be fully specified by $K$ pairs of coefficients $\left(c_{k}, t_{k}\right)$. It was shown that in the exact, noiseless case it is possible to obtain exact reconstruction via $N \geq 2 K$ samples of the filtered signal taken at $t=n T$ by computing the $K$ pairs of coefficients $\left(c_{k}, t_{k}\right)$. Let the sampling kernel be $\varphi(t)$ and the samples obtained from uniformly sampling be $y_{n}$ :

$$
y_{n}=\langle x(t), \varphi(t-n T)\rangle, \quad n=0, \ldots, N-1 \text {. }
$$

Simply speaking, we require that the sampling kernel $\varphi(t)$ allows us to compute $\left\{\left(c_{k}, t_{k}\right)\right\}$ from observations $\left\{y_{n}\right\}_{n=0}^{N-1}$. We say that we have aliasing of an FRI signal when we have undermodeling of the signal.

Almost all of the research in sampling FRI signals relies on solving the following system of equations, called the powersum series:

$$
w_{n}=\sum_{k=0}^{K-1} a_{k}\left(u_{k}\right)^{n}, \quad n=0, \ldots, N-1 .
$$

Given a set of observations $\left\{w_{n}\right\}$, we wish to find $\left\{a_{k}, u_{k}\right\}$. One method of solution in the exact, noiseless case is called the annihilating filter in the signal processing community [2] and was first used to find frequencies of complex harmonics in which $u_{k}=\exp \left(j \omega_{k}\right)$. The other known method of solution is called the rotational invariance technique, which is derived by taking advantage of the special structure of Vandermonde matrices [3]. Both methods first solve for $\left\{u_{k}\right\}$, and then solves the linear system for $\left\{a_{k}\right\}$. The system of equation (2) can also be solved for non-contiguous indices of $n$, as long as there are enough distinct observations available.

For the case when $K=1$ and $N=2$, we have a system of equations with 2 unknowns. Then we can quickly solve $\hat{a}_{0}=w_{0}, \hat{u}_{0}=w_{1} / \hat{a}_{0}$. This is the only instance we know of in which there is a closed-form solution, and one that gives $\hat{a}_{k}$ before $\hat{u}_{k}$. We will take advantage of the relative simplicity of the lower order problems.

In this paper we first review the state-of-the-art of FRI sampling schemes giving local reconstruction, which rely on solving powersums, and then propose new schemes that take samples simultaneously at the outputs of different channels. 
The channels are generated via transformations of the original signal. This is a departure from the previous method of uniform sampling in time.

The work of Vetterli et al. [1] considered the use of sinc, Gaussian, and box kernels. More recently this class of sampling theorems has been extended by Dragotti et al. $[4,5]$ to include functions that which satisfy the Strang-Fix conditions [6], which were first considered in the context of interpolation.

Multi-channel sampling, generalized sampling, and other sampling and reconstruction methods based on linear projections have been considered by many authors [7-10]. However, they only considered filtered versions of the original signal, and drew connections to forming a Riesz basis from the filters. In our work we consider a more general form of transformations, and in departure from the work listed above we consider nonlinear reconstruction methods. Throughout this paper we will focus on a specialized model of (1), in which any finite interval of a certain length contains at most a known number of Diracs.

\section{SAMPLING WITH KERNELS THAT SATISFY THE STRANG-FIX CONDITIONS}

Consider a signal model in which there are at most $K$ Diracs in any interval of length $K C T$, with $C$ and $T$ to be defined later. Although the number of Diracs, and thus the length of the signal, may be infinite, this signal has a finite rate of innovation. In recent work $[4,5]$ it was shown that the class of kernels that can be used for FRI sampling of such signals include those that are compact and able to reproduce polynomials or exponentials. The first condition states that for such a kernel $\varphi(t)$ there must exist $c_{m, n}$ such that $\sum_{n} c_{m, n} \varphi(t-n)=t^{m}$, $m=0,1, \ldots, N$. The coefficients $c_{m, n}$ are easily obtained for the case where we use B-splines [11]. Due to the compact support of the finite-order B-splines we only need to find the coefficients for the terms that overlap the interval of interest.

Now we can move on to the problem of reconstructing an infinite stream of Diracs. Dragotti et al. gave the following theorem.

Theorem 1 (Sampling infinite sums of Diracs [5]). Consider an infinite stream of Diracs and let $\varphi(t)$ be a sampling kernel that can reproduce polynomials of maximum degree $N-1 \geq 2 K-1$ and of compact support $C$. Then the signal is uniquely determined from $N$ samples $y_{n}=\langle x(t), \varphi(t-n T)\rangle$ if and only if there are at most $K$ Diracs in any interval of length $K C T$.

To obtain the proof, it is sufficient to observe that $s[m]=$ $\sum_{n} c_{m, n} y_{n}=\int x(t) t^{m} d t=\sum_{k=0}^{K-1} a_{k} t_{k}^{m}, m=0,1, \ldots, N$ This is again a powersum series (2), and we can find the unknown terms from a sufficient number of observations. To finish the proof, in the case that $x(t)$ contains infinitely many
Diracs, we can reconstruct it exactly if there is only at most $K$ of them within a compact interval of length $K C T$.

\section{SAMPLING USING MULTIPLE CHANNELS}

All the previous research in FRI is based on the idea of sampling uniformly in time; we now propose two schemes that take samples at the output of multiple channels, each of which contain signals that are transformed versions of the original. A trivial modification to the scheme of Dragotti et al. is to parallelize the sampling operation. After all, given a sampling kernel $\varphi(t)$, filtering and uniform sampling in time is simply computing a sequence of observations $\langle x(t), \varphi(t-n T)\rangle$. Obtaining the $N$ samples at the output can be done by simultaneously sampling the output of $N$ filters $F_{n}(t)$ in parallel, given by $F_{n}(t)=\varphi(t-n T)$. Following Dragotti's paper, one possible choice of $\varphi$ is the B-spline. Recall that a B-spline can reproduce polynomials up to its order by taking linear combinations of its shifted versions.

\subsection{Sampling using integrals}

Consider the following set of signals, which are obtained through successive integration:

$$
x_{\ell+1}(t)=\int_{0}^{t} x_{\ell}(\tau) d \tau, \quad x_{0}(t)=\int_{0}^{t} x(\tau) d \tau
$$

We are given samples of these signals at time $T$, denoted as $y_{\ell}=x_{\ell}(T)$. Then the samples can be written as:

$$
y_{\ell}=x_{\ell}(T)=\sum_{k=0}^{K-1} c_{k}\left(T-t_{k}\right)^{\ell-1}, \quad \ell=0,1, \ldots, L-1
$$

While it can be seen that a set of $L \geq 2 K$ measurements will form a full-rank set of equations, it is difficult to solve this computational problem except for the most trivial case $K=$ $1, L=2$ as we showed above. However, (3) is a powersum series of the form (2); hence we can solve for $\left\{t_{k}\right\}$ and $\left\{c_{k}\right\}$. We call the proposed approach the integral sampling system. We summarize our result in the following theorem.

Theorem 2 (Multi-channel Sampling via Integrals). Let the observed signal be $x(t)=\sum_{k=0}^{K-1} c_{k} \delta\left(t-t_{k}\right)$ where $c_{k} \in \mathbb{C}$ and $t_{k} \in[0, T)$. Let $x_{\ell}(t)$ be the l-th integral of $x(t)$. We are given samples $y_{\ell}=x_{\ell}(T)$ for $\ell=0,1, \ldots, L-1$. Then it is possible to perfectly reconstruct $x(t)$ from $\left\{y_{\ell}\right\}_{\ell=0}^{L-1}$ as long as $L \geq 2 K$. The algorithm can also be applied to non-contiguous integral powers of $x(t)$.

Let $s_{\ell}(t)=(1-t)^{\ell} 1_{[0,1)}$. Then we can rewrite the above sampling scheme as a filter-and-sample scheme using the filters $\left\{s_{\ell}(t / T)\right\}_{\ell=0}^{L-1}$. The above operation can be implemented by using a chain of integrators $x_{\ell}(t)=\int_{0}^{t} x_{\ell-1}(\tau) d \tau$ and $x_{0}(t)=\int_{0}^{t} x(\tau) d \tau$. It is also trivial to extend this scheme 
to an infinite-length signal by segmenting, as we obtain measurement from local portions of the signal. In fact, it is easy to vary the lengths of the different segments with different numbers of Diracs in different segments of the signal.

Moreover, the sampling scheme proposed above has a successive refinement property. Suppose that we know that there is a finite but unknown number $K^{*}$ of Diracs within an interval $[0, T)$. We can start by taking 2 samples and reconstructing 1 Dirac, and compare the results with that obtained by taking another 2 samples and and reconstructing 2 Diracs. If the results are consistent, then we stop the sampling operation. Otherwise, having reconstructed $K$ Diracs from $2 K$ samples we take another 2 samples and compare the reconstruction with that for $K+1$ Diracs from the larger set of $2 K+2$ samples. We stop when $K=K^{*}+1$ and the result is consistent with that of the previous reconstruction. In the presence of noise or model mismatch, a stopping criteria other than exact reconstruction can be defined.

\subsection{Sampling using B-splines}

The previous part is related to the idea of using splines for sampling. Let $\beta_{0}(t)$ be the 0 -th order box B-spline, defined as $\beta_{0}(t)=u(t)-u(t-1)$, where $u(t)$ is the usual step function. The higher-order B-splines can be generated recursively as $\beta_{k+1}=\beta_{k} * \beta_{0}$.

Consider the following set of observations:

$$
y_{\ell}=\left\langle\beta_{\ell}(t), x(t)\right\rangle, \quad \ell=0,1, \ldots, L-1 .
$$

Since the first segment of a B-spline is a monomial of the order of the spline, sufficiency is achieved when $L \geq 2 K$ just like in the previous case. It is important to note that the samples above are taken all at the same time, but across different splines scales. This is different from the spline interpolation scheme which takes samples within one spline scale but at different, uniformly spaced time instances. Similarly, the B-spline sampling scheme also has a successive refinement property.

Theorem 3 (Multi-channel Sampling via B-splines.). Let the observed signal be $x(t)=\sum_{k=0}^{K-1} c_{k} \delta\left(t-t_{k}\right)$ where $c_{k} \in \mathbb{C}$ and $t_{k} \in[0, T)$. Without loss of generality, let $T=1$. Further let $y_{\ell}=\left\langle\beta_{\ell}(t), x(t)\right\rangle, \ell=0,1, \ldots, L-1$, the samples taken at time $t=T$ at the outputs of B-spline filters of different orders $\ell$. Then it is possible to perfectly reconstruct $x(t)$ from $\left\{y_{\ell}\right\}_{\ell=0}^{L-1}$ as long as $L \geq 2 K$. It is also possible to solve from $L$ non-contiguous B-spline-filtered samples.

The system proposed above can be implemented by a chain of $\beta_{0}$ filters, similar to the chain of integrators of the previous part. The sampling scheme proposed above can also be applied to signals with different numbers of Diracs at different segments, allowing for lower-order local reconstruction of the signal. However, the knots of the splines are at integer multiples of $T$, and hence the segments must all have the same length.

\subsection{Generalizing sampling based on powersums}

In order to relate our new sampling schemes to the Dragotti et al. scheme we write the previous work in a new notation:

$$
\mathbf{w}=\mathbf{C y}
$$

where $\mathbf{w} \in \mathbb{C}^{M}, \mathbf{y} \in \mathbb{C}^{N}, \mathbf{C} \in \mathbb{C}^{M \times N}$, and $N \geq M$. In the above, $y_{n}=\left\langle x(t), F_{n}(t)\right\rangle$. Clearly, the Dragotti scheme is obtained by settting $F_{n}(t)=\varphi(t-n T)$, and the coefficients $c_{n, m}$ are the same as the ones prescribed by the Strang-Fix conditions. Basically the coefficients are there so that for $x(t)$ in the class of signals composed of $K$ Diracs within an interval $[0, T)$ we will obtain the powersum series (2). In their case the choice of $F_{n}$ is constrained to be of a particular form that represents uniform sampling in time. In general, for example in the cross-scale sampling scheme described above, this constraint is not imposed. The notation above can be used to combine outputs of different kernels sampled at different times, or scales.

The integral-based sampling scheme can be written in this form with $c_{m, n}=\delta_{m, n}$, and $y_{n}=\left\langle x(t), s_{n}(t / T)\right\rangle$. Because $y_{n}$ already looks like the powersum of (2) for this choice of sampling kernel, we do not have to do further processing. Of course, we can elect to if we like. We can take linear combinations of the entries of $\mathbf{y}$ and $\mathbf{C}$ correspondingly to obtain new sampling kernels $F_{n}(t)$.

\section{DETECTING UNDERMODELING AND SUCCESSIVE REFINEMENT VIA MULTICHANNEL SAMPLING}

We have seen that reconstructing 1 Dirac in a finite interval using integral-based sampling can be done using a comparatively simple computation. If we are able to partition a signal $x(t)$ into segments in which there are at most 1 Dirac in each segment, then we can use the simpler algorithm on each segment, taking 2 samples in each segment. Consider $P$ such consecutive segments $\mathcal{S}_{p}=\left[T_{p}, T_{p+1}\right)$, yielding say signal model A. A more general signal model is one which contains $P$ Diracs anywhere within the union of those segments, say $[0, T)$, which we call signal model B. In both cases we have to take at least $2 P$ samples, but these samples are not the same for both cases.

Signal model A is more restrictive than $\mathrm{B}$, but gives a simpler reconstruction formula. In multichannel sampling it is possible to detect undermodeling, for example when we mistakenly assume signal model $\mathrm{A}$. Consider the reconstruction algorithms for models $\mathrm{A}$ and $\mathrm{B}$ respectively:

1. System A: Obtain samples $y_{0}[p]=\int_{\mathcal{S}_{p}} x(t) d t, y_{1}[p]=$ $\int_{\mathcal{T}_{p}}^{T_{p+1}} \int_{\mathcal{T}_{p}}^{t} x\left(t_{1}\right) d t_{1} d t$. From each $p$ use $y_{0}[p]$ and $y_{1}[p]$ to compute estimates $\hat{c}_{p}, \hat{t}_{p}$.

2. System B: Obtain samples $z_{\ell}=\left\langle x(t), s_{\ell}(t / T)\right\rangle$ the $\ell$ th integral of $x(t)$ sampled at $t=T$, for $\ell=0, \ldots, 2 P$ 
1. Use these samples to simultaneously obtain estimates $\left\{\hat{c}_{p}, \hat{t}_{p}\right\}_{p=0}^{P-1}$.

Let $\left\{c_{p}, t_{p}\right\}$ be the coefficients of the Dirac in segment $\mathcal{S}_{p}$. When there is no undermodeling we can derive $\left\{z_{\ell}\right\}$ from $\left\{y_{0}[p], y_{1}[p]\right\}$ by first computing estimates $\left\{\hat{c}_{p}, \hat{t}_{p}\right\}$. From these estimates we can compute an estimate of what the samples of System B should be based on the samples of System A which we call $\left\{\hat{z}_{\ell}\right\}$. We give the following lemma.

Lemma 4. Consistency for lower-order estimates: Consider the two systems $A$ and $B$ defined above, giving samples $\left\{y_{0}[p], y_{1}[p]\right\}$ and $\left\{z_{\ell}\right\}$ respectively. We say that these samples are consistent when we can derive the latter from the former via signal parameter estimation. Let $\left\{\hat{c}_{p}, \hat{t}_{p}\right\}$ be the signal parameter estimates from samples of System $A$, and let $\left\{\hat{z}_{\ell}\right\}$ be the estimate of what the samples of System B should be, given the parameter estimates from $A$. Then for $\ell=0,1$ we always have consistency, whereas for $\ell \geq 2$ the samples of the two systems are consistent only if there is no undermodeling.

Proof. Consider signal model A, and without loss of generality let every segment have 1 Dirac except for segment- $a$ which has two Diracs. We denote this Dirac $\left\{c_{u}, t_{u}\right\}$. Then for $p \neq a$ the estimates obtained from $\mathrm{A}$ are correct, but for $p=a$ we have that $\hat{c}_{a}=c_{a}+c_{u}, \hat{t}_{a}=T_{a}-\left(c_{a}\left(T_{a+1}-\right.\right.$ $\left.\left.t_{a}\right)+c_{u}\left(T_{a+1}-t_{u}\right)\right) /\left(c_{a}+c_{u}\right)$. Since $\hat{z}_{\ell}=\sum_{p} \hat{c}_{p}\left(T-\hat{t}_{p}\right)^{\ell}$, we have that $z_{0}=\hat{z}_{0}$ and $z_{1}=\hat{z}_{1}$, but $z_{\ell} \neq \hat{z}_{\ell}$ for $\ell \geq 2$.

Theorem 5 (Detecting aliasing in low-order FRI signals:). Let $\mathcal{S}_{p}, p=0, \ldots, P-1$ be a P-segmentation of a signal $x(t)$ such that we expect at most 1 Dirac in each segment. From each segment, we compute $y_{0}[p]=\int_{\mathcal{S}_{p}} x(t) d t$ and $y_{1}[p]=\int_{\mathcal{T}_{p}}^{T_{p+1}} \int_{\mathcal{T}_{p}}^{t} x\left(t_{1}\right) d t_{1} d t$, from which we can compute the estimate of one Dirac in each $\mathcal{S}_{p}$. Then it is possible to detect aliasing in any of the $P$ segments by taking one additional sample from a higher-order integral of the signal.

The theorem follows immediately from Lemma 4 . The lemma also suggests that we can "recycle" the samples already taken from sytem $A$ for reconstruction based on samples taken from system $B$, which admits a less restrictive signal model. Finally, we note that these properties also apply to sampling with B-splines.

\section{CONCLUSION}

We have shown that it is possible to perfectly reconstruct signals consisting of a sum of Diracs across multiple channels, for example using simple operations such as integrate-anddump and using a chain of simple filters. These schemes have a successive refinement property, which can be used in the case where we have an unknown, but finite number of Diracs within a finite interval. Further, we showed that it is possible to detect aliasing in certain cases. This is especially useful because more restrictive signal models in which there is only one Dirac in a given segment can be solved easily compared to the more general model in which there are many Diracs in a given finite interval.

\section{REFERENCES}

[1] M. Vetterli, P. Marziliano, and T. Blu, "Sampling signals with finite rate of innovation," IEEE Transactions on Signal Processing, vol. 50, no. 6, pp. 1417-1428, June 2002.

[2] P. Stoica and R. Moses, Spectral Analysis of Signals, Prentice-Hall, Upper Saddle River, NJ, 2005.

[3] R. Roy and T. Kailath, "ESPRIT - Estimation of Signal Parameters via Rotational Invariance Techniques," IEEE Transactions on Acoustics, Speech, and Signal Processing, vol. ASSP-37, pp. 984-995, July 1989.

[4] P. L. Dragotti and M. Vetterli, "Wavelet and footprint sampling of signals with a finite rate of innovation," in IEEE Conference on Acoustics, Speech and Signal Processing, Montreal, Canada, May 2004, vol. 2, pp. 941944.

[5] P. L. Dragotti, M. Vetterli, and T. Blu, "Exact sampling results for signals with finite rate of innovation using Strang-Fix conditions and local kernels," in Proc. IEEE Conf. on Acoustics, Speech, and Signal Proc., Philadelphia, PA, March 2005

[6] G. Strang and G. Fix, A Fourier Analysis of the Finite Element Method, pp. 793-840, C.I.M.E., Rome, 1973.

[7] A. Papoulis, Signal Analysis, McGraw-Hill, New York, 1977.

[8] A. Papoulis, "Generalized sampling expansion," IEEE Trans. Circuits and Systems, vol. CAS-24, no. 11, pp. 652-654, November 1977.

[9] J. R. Higgins, Sampling Theory in Fourier and Signal Analysis, Oxford Science Publications, Oxford, UK, 1996.

[10] M. Unser, "Sampling-50 years after Shannon," Proceedings of the IEEE, vol. 88-4, pp. 569-587, April 2000.

[11] M. Unser, "Splines: A perfect fit for signal and image processing," IEEE Signal Proc. Magazine, pp. 22-38, November 1999. 